\title{
Effect of electrolyzed water produced using carbon electrodes on HeLa cell proliferation
}

\author{
Kyoko Nakamura, ${ }^{1, *}$ Osamu Muraoka ${ }^{1,2}$ \\ 'Pharmaceutical Research and Technology Institute, Kindai University, Osaka, Japan; \\ ${ }^{2}$ Antiaging Center, Kindai University, Osaka, Japan.
}

\begin{abstract}
Summary We developed electrolyzed water (EW) using carbon electrodes and investigated the ability of the developed EW to inhibit the proliferation of human cervical carcinoma HeLa cells. We observed that EW-containing media inhibited HeLa cell proliferation. Many very small black dots were produced in EW and these were associated with the inhibitory effect on the cell proliferation. Furthermore, the very small black dots that could inhibit cell proliferation were produced only at $\mathrm{pH} 3$ to $3.5 \mathrm{of} \mathrm{EW}$. Additional experiments showed that this inhibition of proliferation is reversible. These results suggest that the effect of EW on HeLa cells is cytostatic and not cytotoxic. Thus, our results indicate that the EW developed in this study may be used to inhibit cell proliretation.
\end{abstract}

Keywords: Electrolyzed water, carbon electrodes, cell proliferation

\section{Introduction}

Electrolyzed water is produced by the electrolysis of ordinary tap water or any liquid water containing dissolved electrolytes, such as sodium chloride. During the electrolysis process, a membrane partition (diaphragm) between the anode and cathode results in water being electrolyzed and the production of acidic water at the anode and alkaline water at the cathode. Various types of electrolyzed water can be produced in electrolytic cells, with or without diaphragm, using different kinds of electrolytes. Electrolyzed water is useful in various fields such as in medicine, and in agricultural and food industries $(1,2)$. Electrochemically oxidized or reduced water is produced in an electrolysis chamber with a diaphragm, with electrochemically oxidized water being produced in the anode chamber. In particular, strongly acidic water $(\mathrm{pH} \leq 3)$ has been used as sterile, antibacterial water for sanitizing food contact surfaces and equipment, such as cookware and glasses. Strongly acidic water $(\mathrm{pH}<2.7)$ is

Released online in J-STAGE as advance publication December 11, 2017.

*Address correspondence to:

Dr. Kyoko Nakamura, Pharmaceutical Research and Technology Institute, Kindai University, 3-4-1 Kowakae, Higashiosaka, Osaka 577-8502, Japan.

E-mail:kyoko@phar.kindai.ac.jp processed by electrolysis through a dilute sodium chloride solution and has a strong bactericidal effect on bacteria, virus and fungi (3). Furthermore, Nishida et al. reported that the gargling with this water can inhibit plaque formation (4). Hypochlorous acid in this water is one of the active factors responsible for the bactericidal effect (5). In contrast, electrochemically reduced water is produced in cathode chamber, which has an alkaline $\mathrm{pH}$. Potable electrochemically reduced water $(\mathrm{pH} 8-10)$ is called alkali-ionic water and is popular "health water" in Japan (2). Electrochemically reduced water has been shown to exert anti-cancer effects. For example, electrochemically reduced water produced near the cathode during the electrolysis of ultrapure water containing sodium hydroxide at $100 \mathrm{~V}$ for $60 \mathrm{~min}$ using an electrolyzing device equipped with platinum-coated titanium electrodes suppressed tumor angiogenesis in human lung adenocarcinoma A549 cells and tumor invasion in human fibrosarcoma HT1080 cells $(6,7)$. Recently, several researchers have reported the generation of electrolyzed water of neutral $\mathrm{pH}$ by an electrolysis chamber without a diaphragm. Neutral $\mathrm{pH}$ hydrogen-enriched electrolyzed water inhibited efficient colony formation of human tongue squamous cell carcinoma-derived HSC-4 cells and growth of human fibrosarcoma HT-1080 cells (8). Slightly acidic electrolyzed water ( $\mathrm{pH} 5.0$ to 6.5 ) produced in an electrolysis chamber without a diaphragm containing a $2 \%$ to $6 \%$ hydrogen chloride solution exhibited 
antibacterial effects on oral pathogens and inhibited the proliferation of human gingival fibroblasts (9). Therefore slightly acidic electrolyzed water is usually used as a disinfectant.

Thus, various types of electrolyzed water are beneficial for human health. Particularly, we focused on the inhibitory effect of electrolyzed water against cancer cells. Here, we produced electrolyzed water (EW) from tap water in an electrolysis chamber without a diaphragm using special carbon electrodes. We have assessed the effect of our EW on tumor cell proliferation. Our results demonstrate that EW generated using special carbon electrodes inhibits the proliferation of human cervical carcinoma HeLa cells.

\section{Materials and Methods}

\subsection{Preparation of electrolyzed water (EW) using special carbon electrodes}

We used special carbon electrodes $(150 \times 30 \times 7 \mathrm{~mm})$ prepared by Toyo Tanso Co. Ltd. (Osaka, Japan) and these electrodes were graphite-resin composite electrodes. It was known that electrolysis of water using these electrodes produced amount of aqueous carbon dioxide. EW was prepared by electrolysis of tap water (W) at $10 \mathrm{~V}$ for $72 \mathrm{~h}$ in a $1,000 \mathrm{~mL}$ beaker equipped with special carbon electrodes. In the electrolysis process, two carbon electrodes were set in $\mathrm{V}$-formations and EW was a mixture of anode-side and cathodeside electrolyzed water. Both electrodes need to be immersed to length of about $10 \mathrm{~cm}$ into water. Figure $1 \mathrm{~A}$ depicts a schematic diagram of water electrolysis. We assume that carbon compounds $\left(\mathrm{C}_{\mathrm{x}} \mathrm{H}_{\mathrm{O}} \mathrm{O}_{\mathrm{z}}\right)$ were produced in the resulting EW. We confirmed that the mass of $\mathrm{C}_{\mathrm{x}} \mathrm{H}_{\mathrm{y}} \mathrm{O}_{\mathrm{z}}$ was 2,000 and 4,000 Da by laser TOF Mass analysis (Toyo Tanso Co. Ltd.) of EW for the molecular characterization of $\mathrm{C}_{\mathrm{x}} \mathrm{HyO}_{\mathrm{z}}$ (data not shown). The EW turned light brown and had a $\mathrm{pH}$ of 3 to 3.5 . On the other hand, the control (W) was colorless, transparent, and had a $\mathrm{pH}$ of around 7.

\subsection{Cell culture and preparation of EW-containing media}

HeLa cells were plated onto culture dishes and cultured in low glucose Dulbecco's modified Eagle medium (DMEM, Sigma, St. Louis, MO, USA) supplemented with $10 \%$ fetal bovine serum (FBS) (Nichirei Biosciences Inc., Tokyo, Japan), $100 \mu \mathrm{g} /$ $\mathrm{mL}$ streptomycin, and $100 \mathrm{U} / \mathrm{mL}$ penicillin (Nacalai Tesque, Kyoto, Japan). Cells were maintained at $37^{\circ} \mathrm{C}$ in a humidified atmosphere containing $5 \% \mathrm{CO}_{2}$ and $95 \%$ air.

In order to investigate the effects of EW on the proliferation of HeLa cells, media were prepared using EW or W (control) instead of ultra-pure water. The

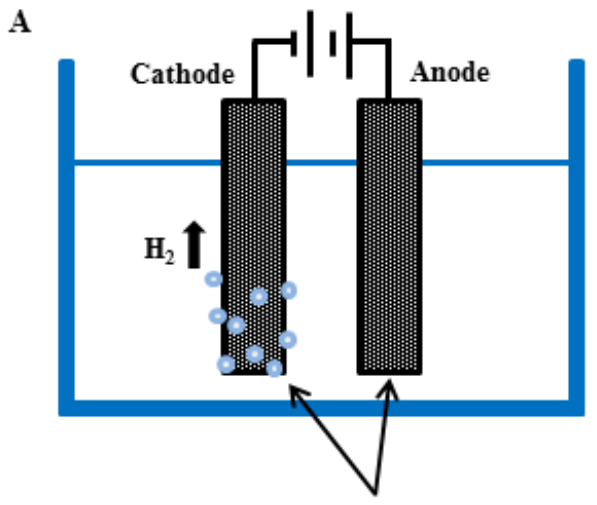

Special carbon electrodes

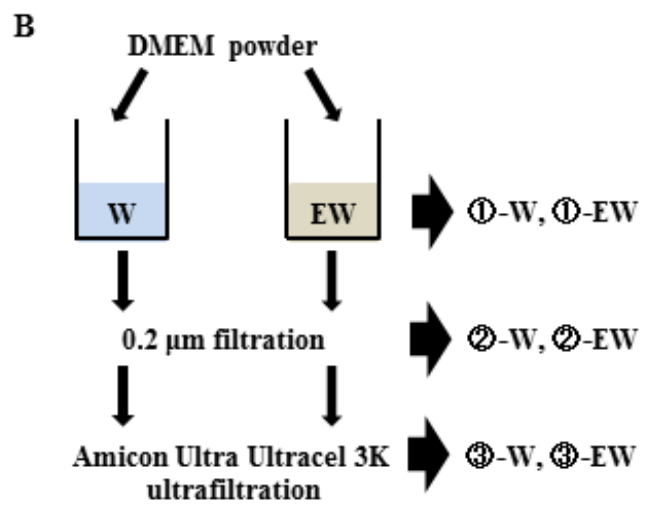

Figure 1. Schematic view of EW generation system and preparation of culture media. A. Principle of EW preparation. The chemical reaction is follows:

Anode side

$\mathrm{H}_{2} \mathrm{O} \rightarrow 2 \mathrm{H}^{+}+\mathrm{O}^{\cdot}+2 \mathrm{e}^{-}$

$\mathrm{C}+2 \mathrm{O}^{*} \rightarrow \mathrm{CO}_{2}$ (aq)

$\mathrm{C}+\mathrm{O}^{\circ}+\mathrm{H}_{2} \mathrm{O} \rightarrow \mathrm{C}_{\mathrm{X}} \mathrm{H}_{\mathrm{Y}} \mathrm{O}_{\mathrm{Z}} \mathrm{C}_{\mathrm{X}} \mathrm{H}_{\mathrm{Y}} \mathrm{O}_{\mathrm{Z}}$ : the expected molecular weight of 2,000 4,000

Cathode side

$2 \mathrm{H}^{+}+2 \mathrm{e}^{-} \rightarrow \mathrm{H}_{2}$

B. Schematic flow diagram representing the preparation of various media. Add DMEM powder to $\mathrm{W}$ or EW with gentle stirring (1)-W or (1)-EW). Filtration through $0.2-\mu \mathrm{m}$ filter of (1) $-W$ or (1)-EW (2)-W or (2)-EW). Furthermore, (2)-W or (2)-EW is filtered using Amicon Ultra-15 3K centrifugal filter units (3-W or (3-EW).

preparation of various media used in this experiment are shown in Figure 1B. First, DMEM powder (Nissui Pharmaceutical Co., Ltd., Tokyo, Japan) was dissolved in W or EW (1)-W or (1)-EW) according to manufacturer's instruction. Then, these media were filtered through a $0.2-\mu \mathrm{m}$ filter (cellulose acetate membrane) (2)-W, (2)-EW). Furthermore, (2)-W and (2)-EW were each filtrated using Amicon Ultra-15 $3 \mathrm{~K}$ centrifugal filter units (low-binding regenerated cellulose membrane) (Uitracel-3K, Millipore Co. Ltd., Billerica, MA, USA) (3-W, (3)-EW). The (1)-EW turned brownish-yellow and was somewhat turbid, which many differently sized black dots were visible. The (2)-EW turned weakly brownish-yellow and was a little turbid, which very small black dots had precipitated to the bottom after being left standing for a few days. The (3)-EW turned yellow and exhibited relatively low turbidity, which very small black dots did not observed 
under the microscope. This process generated 6 types of media (1)-W, (1)-EW, (2)-W, (2)-EW, (3)-W and (3)-EW) that were each sterilized by autoclaving at $121^{\circ} \mathrm{C}$ for $15 \mathrm{~min}$ and then supplemented with $10 \% \mathrm{FBS}, 0.584$ $\mathrm{g} / \mathrm{L}$ glutamine, $100 \mu \mathrm{g} / \mathrm{mL}$ streptomycin, $100 \mathrm{U} / \mathrm{mL}$ penicillin, and $10 \%$ sodium hydrogen carbonate. The final average $\mathrm{pH}$ values were as follows: (1)-W, 8.41; (1)-EW, 8.21; (2)-W, 8.24; and (2)-EW, 8.05. EW media had a slightly lower $\mathrm{pH}$ than that of $\mathrm{W}$ media, but all media were still alkaline. Filtration of media using Amicon Ultra-15 3K centrifugal filter units had no effect on $\mathrm{pH}$ (data not shown).

\subsection{Cell proliferation and EW washout assay}

After removing the culture media, the HeLa cells grown in culture dishes were washed with sterile PBS (phosphate buffered saline) (137 mM sodium chloride, $8.1 \mathrm{mM}$ sodium phosphate dibasic, $2.7 \mathrm{mM}$ potassium chloride, and $1.47 \mathrm{mM}$ potassium phosphate monobasic) and detached with $0.25 \%$ trypsin-EDTA. HeLa cells were collected by centrifugation at $800 \times \mathrm{g}$ for $5 \mathrm{~min}$ and resuspended in each type of culture media (1)-W, (1)-EW, (2)-W, (2)-EW, (3)-W and (3)-EW). HeLa cells were seeded onto culture flasks at a density of 8 $\times 10^{4}$ cells/flask and incubated for 3 days at $37^{\circ} \mathrm{C}$ in a humidified atmosphere containing $5 \% \mathrm{CO}_{2}$ and $95 \%$ air. The effect of EW on cell proliferation was then assessed by counting the number of viable cells. HeLa cells were trypsinized, stained with trypan blue, and counted in a hemocytometer every day for 3 days.

The reversibility of the inhibition of cell proliferation after EW washout was assessed in an additional study by replacing EW media with $\mathrm{W}$ control media after 2 days. HeLa cells cultured in (2)-EW medium for 2 days were washed twice with PBS and resuspended in (2)-W control or (2)-EW medium. HeLa cells were trypsinized, stained with trypan blue, and counted in a hemocytometer every day for additional 3 days.

\section{Results and Discussion}

To investigate the effects of EW-containing media on tumor cell proliferation in human cervical carcinoma HeLa cells, HeLa cells were first cultured in (1)-W (control) or (1)-EW media for 3 days. Figure 2A and $2 \mathrm{~B}$ are photographs of HeLa cells after a day of culture in (1)-W (control) (Figure 2A) or (1)-EW (Figure 2B) media. We observed differently sized black dots in the (1)-EW medium under the microscope (Figure 2B). We speculate that these black dots were broken pieces of carbon (Figure 2B) from the carbon electrode used in water electrolysis. These black dots were not observed in the (1)-W (control) medium (Figure 2A). Interestingly, HeLa cell proliferation was markedly inhibited by incubation in cell culture media containing
A

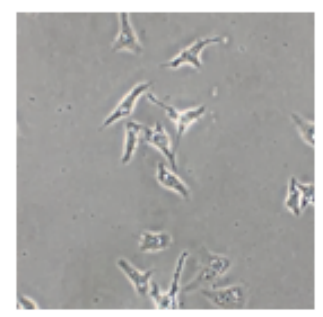

(1)-W
B

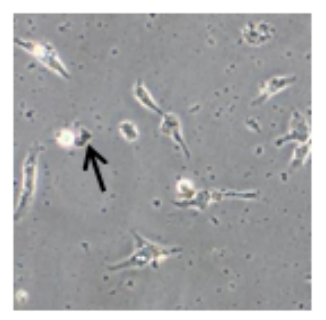

(1)-EW

C

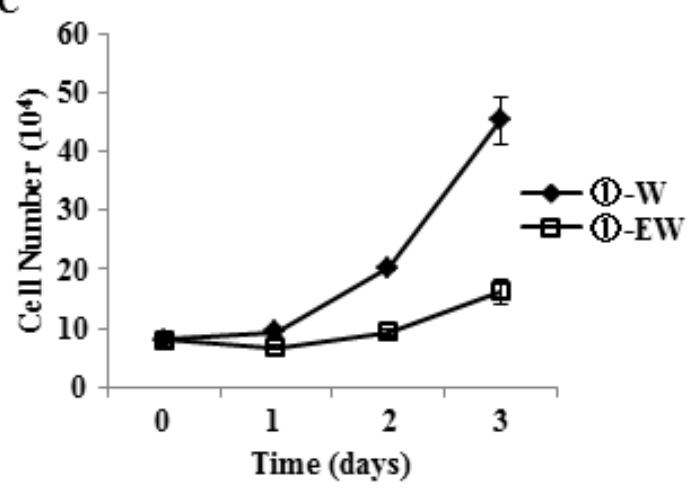

Figure 2. The effect of (1)-W and (1)-EW media on proliferation of HeLa cells. Cells were seeded at a density of $8 \times 10^{4}$ cells in flasks containing each culture medium. After 1 day of incubation, cells were photographed at $\times 10(\mathbf{A}, \mathbf{B})$ and then counted daily for 3 days $(\mathbf{C})$. The arrow indicates a conceivable broken piece of carbon electrode. Data are expressed as the mean $\pm \mathrm{SD}$ of three independent experiments.

EW compared to those containing W (control) (Figure $2 \mathrm{C})$. These results demonstrate that EW-containing medium has the potential to inhibit the proliferation of HeLa cells.

In this study, EW was prepared by electrolysis of ordinary tap water. Although it was conceivable that hypochlorous acid might be produced by electrolysis of chlorine ions in tap water, EW generated in this study weakly contained hypochlorous acid at very low chlorine level $(0.00325 \mathrm{ppm})$ and culture media for HeLa cells prepared using EW were sterilized by autoclaving at $121^{\circ} \mathrm{C}$ for $15 \mathrm{~min}$. Since hypochlorous acid was removed by boiling in culture media, we focused on black dots in cell culture media as an inhibitor of cell proliferation. To test whether these black dots in (1)-EW medium are involved in cell proliferation, we attempted to remove the dots from the medium using a $0.2-\mu \mathrm{m}$ filter. As shown in Figure $3 \mathrm{~B}$, the relatively large black dots in (2)-EW medium could be removed using a $0.2-\mu \mathrm{m}$ filter. However, very small black dots were still observed in (2)-EW medium (Figure 3B), indicating that there did not adsorbed onto $0.2-\mu \mathrm{m}$ filter membrane. (2)-EW medium still retained the ability to inhibit cell proliferation to the same extent as (1)-EW (Figure 2C and 3C). (1)-W (control) medium was also filtered in the same way as that for (2)-W medium (Figure $3 \mathrm{~A}$ ); this filtration had no effect 
A

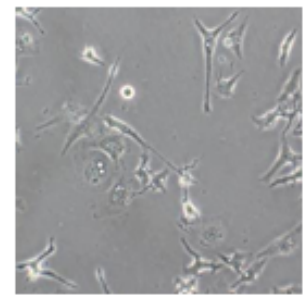

(2)-W

C
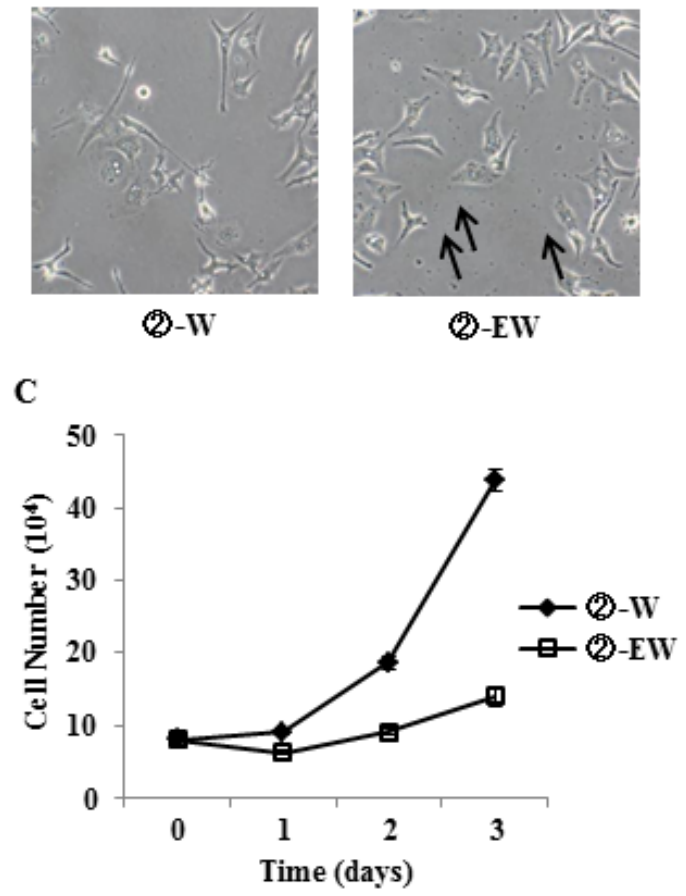

(2)-EW
D

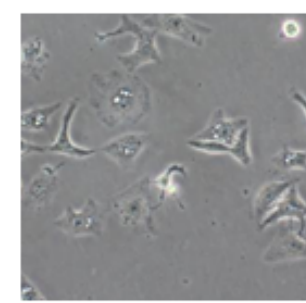

(3)-W
$\mathbf{E}$

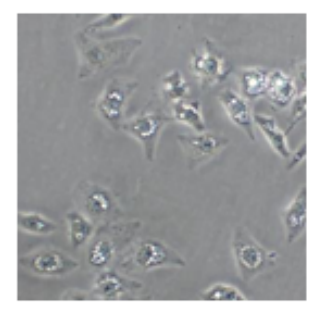

(2)-EW

F

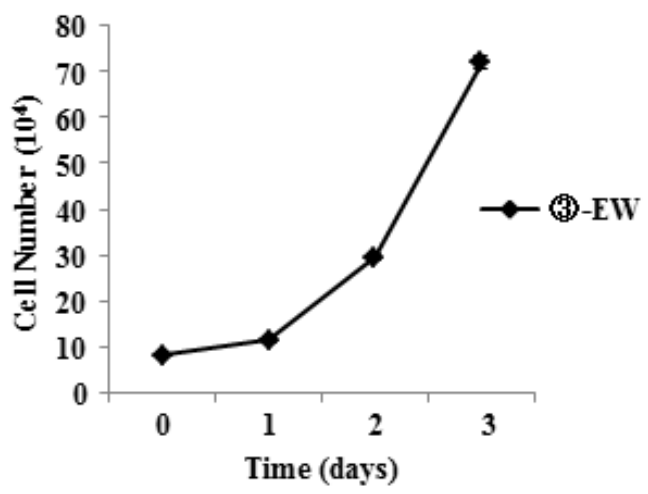

Figure 3. The effect of (2)-W or (2)-EW and $\circ$ 3-EW media on proliferation of HeLa cells. Each (2)-W and (2)-EW was prepared by filtration $(0.2 \mu \mathrm{m})$ of (1)-W and (1)-EW, respectively. Cells were seeded at a density of $8 \times 10^{4}$ cells in flasks containing each culture medium. After 1 day of incubation, cells were photographed at $\times 10(\mathbf{A}, \mathbf{B})$ and counted every day for 3 days $(\mathbf{C})$. The arrows indicate very small black dots. Each (3-W and (3)-EW was prepared by centrifugation of (2)-W and (2)-EW in Amicon Ultra-15 3K centrifugal filter units. Cells were seeded at a density of $8 \times 10^{4}$ cells in flasks containing each culture medium. After 1 day of incubation, cells were photographed at $\times 10(\mathrm{D}, \mathrm{E})$ and then counted for 3 days (F). Filtration of Amicon Ultra-15 3K centrifugal filter units had no effect on cell proliferation (data not shown). Data are expressed as the mean \pm SD of three independent experiments.

on cell proliferation (Figure 2C and 3C). Thus, these results indicate that the very small black dots that passed through the $0.2-\mu \mathrm{m}$ filter were associated with significant reduction of cell proliferation. Sometimes, $\mathrm{EW}$ with a $\mathrm{pH}$ greater than 3.5 was generated. Many very small black dots were observed in EW with a $\mathrm{pH}$ greater than 3.5 by microscopy. Surprisingly, cell culture medium containing EW with at a $\mathrm{PH}$ greater than 3.5 was unable to inhibit HeLa cell proliferation (data not shown). We found that the very small black dots that could inhibit cell proliferation were produced only at $\mathrm{pH}$ of 3 to 3.5 . Therefore, the $\mathrm{pH}$ of EW is very important for the inhibition of cellular proliferation.

Next, to remove these black dots involved in inhibiting tumor cell proliferation from (2-EW medium, we used ultrafiltration membranes with much smaller pore sizes, between 0.001 and $0.1 \mu \mathrm{m}$. Ultrafiltration membranes are typically classified by the nominal molecular weight limit (NMWL). Since the predicted molecular weights of carbon compounds $\left(\mathrm{C}_{\mathrm{x}} \mathrm{H}_{\mathrm{y}} \mathrm{O}_{\mathrm{z}}\right)$ produced in EW were between 2,000 and 4,000 Da, the (2)-EW was centrifuged using Amicon Ultra-15 $3 \mathrm{~K}$ centrifugal filter units ( $3 \mathrm{kDa}$ cutoff) (Millipore) and this solution was designated as (3-EW. A similar treatment was performed for (2)-W control to produce (3)-W, and the very small black dots were efficiently removed in (3-EW medium as clear as (3-W medium (Figure 3D and 3E). Surprisingly, removing these black
A

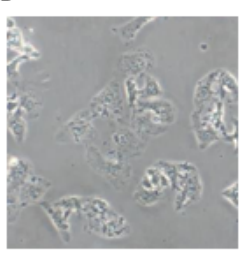

(2) $-\mathrm{EW} \rightarrow$ (2) $-\mathrm{W}$

C

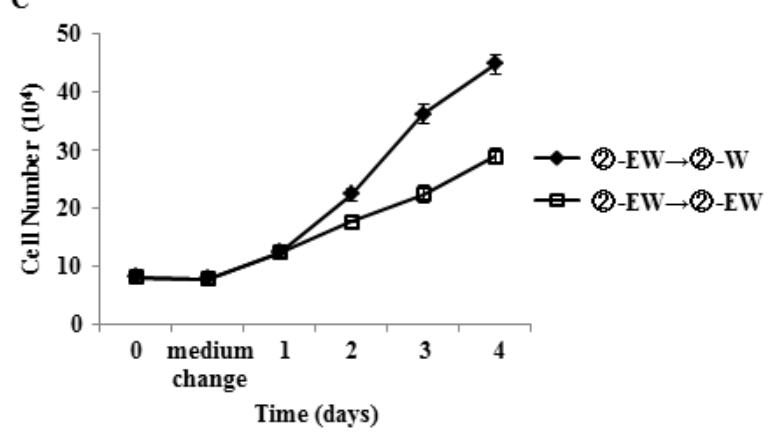

Figure 4. Reversibility of the inhibitory effect of (2)-EW on the proliferation of HeLa cells. Cells were seeded at a density of $8 \times 10^{4}$ cells in flasks containing (2)-EW medium and counted for 2 days. After 2 days of incubation, cells were washed with PBS and reincubated with (2)-W control or (2) -EW medium for more 3 days. Cells were photographed a day after medium replacement at $\times 10(\mathbf{A}, \mathbf{B})$ and then counted for more 3 days (C). The arrows indicate very small black dots and the arrowheads indicate many very small black dots bind to cell surface. Data are expressed as the mean \pm SD of three independent experiments. 
dots from EW recovered the ability of HeLa cells to proliferate in the media (Figure 3F). Cell proliferation rates showed no change in the (3)-W medium (data not shown), indicating that centrifugation using Amicon Ultra-15 $3 \mathrm{~K}$ centrifugal filter units ( $3 \mathrm{kDa}$ cutoff) had no influence on cell proliferation in HeLa cells. Thus, these results indicate that black dots in EW can inhibit proliferation of HeLa cells and can be removed using Amicon Ultra-15 3K centrifugal filter units. Therefore, we suggest that the very small black dots produced in EW have the ability to inhibit tumor cell proliferation and exhibit molecular weights ranging from about 3,000 Da to a molecular weight small enough to enable the dots to pass through a $0.2-\mu \mathrm{m}$ filter.

To determine whether inhibition of HeLa cell proliferation by EW-containing media was a reversible phenomenon, we assessed whether HeLa cells cultured in (2)-EW medium would regain the capacity to proliferate when the medium is replaced with (2)-W control medium. HeLa cells cultured in (2)-EW medium for 2 days were washed twice with PBS to remove black dots, followed by replacement of the medium with (2)-W control medium or (2)-EW medium. As shown in Figure $4 \mathrm{~A}, \mathrm{PBS}$ washes were able to decrease the number of black dots in the flasks containing the replacement (2)-W medium. In contrast, a number of black dots were observed in the flasks containing the replacement (2)-EW medium and some of them were binding to cell surface (Figure 4B). Since the cell surface of many lipid bilayers had negative charges, small black dots might be electrostatically bounded to cell surface. Removal of (2)-EW medium (black dots) by PBS washing resulted in acceleration of the growth rate of HeLa cells (Figure $4 \mathrm{C})$. These results suggest that the inhibitory effect on cell proliferation by EW is reversible, and the black dots expected to be associated with the inhibition of cell proliferation reversibly stuck on the cell surface. These results further indicate that the anti-proliferative effect of EW is not due to a cytotoxic effect and can be stopped by washing twice with PBS.

Growth factors are important for regulating cell proliferation. Growth factors bind to cell surface receptors to initiate signaling pathways that result in the cell proliferation. Insulin-like growth factor 1 (IGF1 ) is one of the major regulators of cell proliferation (10). Upon IGF-1 binding, the tyrosine kinase activity of IGF-1 receptor (IGF-1R) leads to downstream signal activation of the mitogen-activated protein kinase and phosphoinositide 3-kinase-Akt pathways, which promote cell proliferation (11). Furthermore, IGF$1 \mathrm{R}$ plays a central role in the development of many types of tumors $(12,13)$. In our study, we investigated the proliferation of HeLa cells in presence of FBS. Introduction of the monoclonal antibody alpha-IR3 against human IGF-1R reduces proliferation of human non-autocrine neuroblastoma cells SK-N-SH and LF in the presence of FBS (14). Thus, we hypothesize that carbon compounds produced in EW may inhibit cell proliferation by suppressing the signaling pathway for IGF-1R cell proliferation. Future studies are needed to test our hypothesis and to elucidate the mechanism of the anti-proliferation effect of EW in HeLa cells. Furthermore, the electrostatic potential of IGF-1R is overwhelmingly negative (15). The C domain of IGF-1 is electropositive and important for its receptor binding activity (16). Therefore, it is possibility that carbon compounds electrostatically inhibit the IGF-1 binding to IGF-1R and we hope elucidate this possibility in further studies.

In conclusion, we developed EW from tap water using special carbon electrodes and this EW was containing carbon compounds observed very small black dots with molecular weights 2,000 and 4,000 Da. We found that the developed EW inhibited HeLa cell proliferation and that carbon compounds were associated with this significant inhibition of tumor cell proliferation. We also found that washing the cells with PBS could inactivate the inhibitory effect of EW, indicating that there was reversible association between HeLa cells and certain carbon compounds produced in EW and the inhibitory effect of EW was not due to a cytotoxic effect. These findings suggest a novel mechanism for EW in the inhibition of tumor cell proliferation.

\section{Acknowledgements}

The authors thank Mr. Yoshihiro Kojima and Dr. Tetsuro Tojo (Toyo Tanso Co., Ltd., Osaka, Japan) for providing special carbon electrodes.

\section{References}

1. Al-Haq MI, Sugiyama J, Isobe S. Applications of electrolyzed water in agriculture and food industries. Food Sci Technol Res. 2005; 11:135-150.

2. Shirahata S, Hamasaki T, Teruya K. Advanced research on the health benefit of reduced water. Trends food sci technol. 2012; 23:124-131.

3. Okuda R, Sasazaki H, Kanehira M, Okabe T, Abe S, Tagami A, Iwamatsu Y, Miya Y, Shimizu Y. Bactericidal effect of high oxidation potential water viewed from a morphological change. Japanese Journal of Conservative Dentistry. 1994; 37:755-765. (in Japanese)

4. Nishida T, Eda M, Shimada K, Yamada K, Ito K, Murai S. Effects of Acid Electrolysis Water on Plaque Formation. Nihon Shishubyo Gakkai Kaishi. 1993; 35:692-697. (in Japanese)

5. Shiba A, Murai S, Amagasa T. Electrolyzed acidic water in the dental clinic. Tokyo: Quintessence. 1997:35-38.

6. Ye J, Li Y, Hamasaki T, et al. Inhibitory effects of electrolyzed reduced water on tumor angiogenesis. Biol Pharm Bull. 2008; 31:19-26.

7. Kinjo T, Ye J, Yan H, Hamasaki T, Nakanishi H, Toh K, Nakamichi N, Kabayama S, Teruya K, Shirahata S. Suppressive effects of electrochemically reduced water on matrix metalloproteinase- 2 activities and in 
vitro invasion of human fibrosarcoma HT1080 cells. Cytotechnology. 2012; 64:357-371.

8. Saitoh Y, Okayasu H, Xiao L, Harata Y, Niwa N. Neutral $\mathrm{pH}$ hydrogen-enriched electrolyzed water achieves tumorpreferential clonal growth inhibition over normal cells and tumor invasion inhibition concurrently with intracellular oxidant repression. Oncol Res. 2008; 17:247-255.

9. Nakamura T, Oda H, Sato S. Effects of slightly acidic electrolyzed water on oral pathogens and human gingival fibroblasts. Jpn J Conserv Dent. 2010; 53:570-578.

10. Valentinis B, Baserga R. IGF-1 receptor signaling in transformation and differentiation. Mol Pathol. 2001; 54:133-137.

11. Adams TE, Epa VC, Garrett TP, Ward CW. Structure and function of the type 1 insulin-like growth factor receptor. Cell Mol Life Sci. 2000; 57:1050-1093.

12. Macaulay VM. Insulin-like growth factors and cancer. Br J Cancer. 1992; 65:311-320.

13. Baserga R. The insulin-like growth factor I receptor: A key to tumor growth? Cancer Res. 1995; 55:249-252.

14. Burke TW, Vuk-Pavlović S. Insulin-like growth factor-I is a serum component stimulating growth of human neuroblastoma. In Vitro Cell Dev Biol Anim. 1993; 29A:391-394.

15. Lou M, Garrett TP, McKern NM, Hoyne PA, Epa VC, Bentley JD, Lovrecz GO, Cosqrove LJ, Frenkel MJ, Ward $\mathrm{CW}$. The first three domains of the insulin receptor differ structurally from the insulin-like growth factor 1 receptor in regions governing ligand specificity. Proc Natl Acad Sci U S A. 2006; 103:12429-12434.

16. Gill R, Wallach B, Verma C, Ursø B, De Wolf E, Grötzinger J, Murray-Rust J, Pitts J, Wollmer A, De Meyts P, Wood S. Engineering the C-region of human insulin-like growth factor-1: Implication for receptor binding. Protein Eng. 1996; 9:1011-1019.

(Received August 22, 2017; Revised October 25, 2017; Accepted November 26, 2017) 